Ophthalmologe $2021 \cdot 118: 982-985$ https://doi.org/10.1007/s00347-021-01488-0 Angenommen: 28. Juli 2021 Online publiziert: 20. August 2021 (c) Springer Medizin Verlag $\mathrm{GmbH}$, ein Teil von Springer Nature 2021

\title{
Standardisierung in der Bildgebung umsetzen - Ein Plädoyer für den „Digital Imaging and Communication in Medicine"(DICOM)-Standard in der Augenheilkunde
}

\author{
Peter Mussinghoff' · Karsten Kortüm², $\cdot$ Matthias Gutfleisch $\cdot$ Georg Spital' $~$. \\ Eva Hansmann ${ }^{4}$. Thomas Ach ${ }^{5}$. Sandra Liakopoulos ${ }^{6}$. Nicole Eter ${ }^{7}$. \\ Daniel Pauleikhoff' - Albrecht Lommatzsch' \\ 'Augenzentrum am St. Franziskus-Hospital, Münster, Deutschland \\ ${ }^{2}$ Augenklinik, Uniklinik Ulm, Ulm, Deutschland \\ ${ }^{3}$ Augenarztpraxis Dres. Kortüm MVZ GmbH, Ludwigsburg, Deutschland \\ ${ }^{4}$ Bundesverband Deutscher OphthalmoChirurgen e. V., München, Deutschland \\ ${ }^{5}$ Universitäts-Augenklinik, Universitätsklinikum Bonn, Bonn, Deutschland \\ ${ }^{6}$ Zentrum für seltene Augenerkrankungen, Universitätsklinikum Köln, Köln, Deutschland \\ ${ }^{7}$ Klinik für Augenheilkunde, Universitätsklinikum Münster, Münster, Deutschland
}

Bildgebende Verfahren in der Augenheilkunde haben sich in den letzten Jahren rasant entwickelt und Diagnostik sowie Therapiekontrolle revolutioniert. So hat die optische Kohärenztomographie (OCT) die Erkenntnisse zur Pathogenese und zum Verlauf zahlreicher Augenerkrankungen deutlich verbessert. Die bildgebungsgestützte Diagnostik und Therapiekontrolle spielen heute eine zentrale Rolle bei der Betreuung unserer Patienten und sind aus dem Alltag in Klinik und Praxis nicht mehr wegzudenken. Neben der OCT gibt es eine Fülle weiterer Bildgebungsmodalitäten und funktioneller Verfahren, die tagtäglich zum Einsatz kommen und die Diagnostik in der Augenheilkunde zu einer multimodalen Diagnostik machen.

Mit der Entwicklung von neuen bildgebenden und diagnostischen Verfahren begeistern die Hersteller ihre Kunden, nämlich uns Augenärzte, regelmäßig. Technologische (Weiter-)Entwicklungen und neue Erkenntnisse bei der Detektion und Analyse von Befunden generieren und die Effektivität von Therapien zum Wohle der Patienten zu überwachen können tragen zu einer raschen Akzeptanz der Techniken auf Anwenderseite bei.

So faszinierend sich die technologische Entwicklung auf der Geräteseite darstellt, so schleppend ist der Fortschritt bei der von allen Ärzten gewünschten Standardisierung der Bildgebung. Immer noch kämpfen Augenärzte in Klinik und Praxis täglich mit der Situation, dass z.B. OCT-Aufnahmen unterschiedlicher Hersteller nicht über eine einheitliche Software betrachtet werden können. Patienten, die eine OCTAufnahme von einem Augenarzt mit in die Klinik bringen, erleben, dass die Daten nicht eingelesen werden können und die Untersuchung wiederholt werden muss. Stattdessen sind auch in 2021 Papierausdrucke zum Befundaustausch immer noch Standard.

Ärzte, die Bilddaten über telemedizinische Vernetzungslösungen austauschen wollen, erleben, dass sehr mühsame Ex- und Importe in die jeweiligen herstellerspezifischen Softwarelösungen vorgenommen werden müssen. Bei der Umsetzung von innovativen Projekten, z. B. bei klinischen Studien, bei klinischen Registern, in der Telemedizin, bei dem 
Hier steht eine Anzeige.

黑 Springer 
Einsatz von künstlicher Intelligenz und Deep Learning, erfahren Ärzte und Wissenschaftler, dass proprietäre Formate der Hersteller Fortschritt und Entwicklung in diesen wichtigen Zukunftsfeldern verlangsamen oder gar verhindern. Selbst die Gerätehersteller, die neben den Geräten auch eigene Image Managementplattformen (z. B. Heyex [HeidelbergEngineering, Heidelberg, Deutschland], Forum [Carl Zeiss Meditec, Jena, Deutschland], Harmony [Topcon Corporation, Tokio, Japan] entwickeln, kämpfen mit der Integration von bildgebenden Verfahren der jeweils anderen Hersteller.

Die Standardisierung in der ophthalmologischen Bildgebung und Diagnostik liegt im Vergleich zu anderen Fächern wie der Radiologie oder Kardiologie damit sehr weit zurück. Der Digitalisierung in der Augenheilkunde fehlt damit ein wichtiges Standbein, zu häufig ist man mit Krücken unterwegs.

Standardisierung in der Augenheilkunde scheint also ein dickes Brett zu sein, das zu bohren viele Jahre beansprucht.

\section{Wo stehen wir mit der digitalen Standardisierung in der Augenheilkunde?}

Das Thema DICOM in der Augenheilkunde wird seit vielen Jahren - maßgeblich durch die American Academy for Ophthalmology (AAO) - angetrieben [1].

DICOM steht für "Digital Imaging and Communication in Medicine". Der Standard hat einen traditionell starken Fokus auf Bildakquisitionsprozesse. In der Radiologie entwickelt, wird der DICOM-Standard auf viele weitere Domänen ausgeweitet. Zunehmend befasst sich DICOM auch ganz allgemein mit der Kodierung von Behandlungsdaten, was auch die maschinenlesbare Kodierung von Messwerten, Kurven und Befunden beinhaltet. Im Standard-Workflow findet sämtliche Kommunikation eines Gerätes - das Abrufen der Auftragsliste, die Statusrückmeldung und die Übertragung der Behandlungsdaten - nach dem DICOM-Standard statt. Somit ist DICOM in Klinik und Praxis unumgänglich und damit von großer Relevanz für Geräte in der Ophthalmologie. Die Vorbilder aus der Radiologie zeigen, dass eine Umsetzung möglich ist und die Versorgung auf Basis internationaler Standards verbessert und effektiver gestaltet werden kann.

Folgende Standards sind bereits für die Augenheilkunde vollständig definiert und können von den Herstellern der Geräte implementiert werden [2]:

- Ultrasound (DICOM Supplement 5),

- Ophthalmic photography devices (DICOM Supplement 91),

- OCT equipment (DICOM Supplement 110),

- Keratometers and autorefractors (DICOM Supplement 130),

- Reporting of macular grid thickness and volume (DICOM Supplement 143),

- Optical biometry devices (DICOM Supplement 144),

- Visual field perimetry testing equipment (DICOM Supplement 146),

- Optic nerve head topography and retinal thickness mapping devices (DICOM Supplement 152),

- Corneal topography equipment (DICOM Supplement 168),

- Wide-field ophthalmology photography devices (DICOM Supplement 173),

- OCT angiography (DICOM Supplement 197),

- Key measurements in Encapsulated PDF (DICOM Correction Proposal 1811).

ObwohI DICOM-Standards für die Ophthalmologie umfangreich definiert wurden, fehlt es noch an der gewünschten konsequenten Umsetzung hin zu Interoperabilität und Konnektivität zwischen Herstellern, ihren Geräten, Plattformen, Applikationen und Softwarelösungen. Angebote von DICOM-Schnittstellen bleiben z. T. weit hinter der Erwartung des Kunden und weit hinter der Definition im Standard zurück. Zu wenig wird die Möglichkeit genutzt, die Öffnung von Schnittstellen und die aktive Umsetzung von Standards als Chance zu sehen. Für die Qualität der Versorgung der Patienten kann ein einzelner digital übermittelter Befund eine große Bedeutung haben.

Der Bildaustausch zwischen einzelnen Akteuren in der Behandlungskette ist vielfach defizitär, weil digitale Prozesse in der sektorübergreifenden Kommunikation, zwischen Ärzten und Kliniken oder operativen Zentren, nicht auf Basis internationaler Standards gestaltet werden können. Dadurch kommt es zu Kommuni- kationsbrüchen an den Schnittstellen, der Papierausdruck und der Patient als „Datenträger" ist noch allzu häufig der Ausweg aus diesem Dilemma. Ohne gute digitale Prozesse, werden aber die Möglichkeiten und Chancen von neuen digitalen Versorgungskonzepten auch nicht auszuschöpfen sein. Auch werden mögliche Anwendungen über das Deutsche Gesundheitsnetz, der Telematikinfrastruktur, nicht erfolgreich sein, wenn es keinen einheitlichen Dateistandard gibt.

Es ist an der Zeit, für Interoperabilität zu sorgen. Wir fordern die Hersteller der ophthalmologischen Bildgebung und Diagnostik auf, konsequent, umfassend und nachvollziehbar den DICOM-Standard umzusetzen und ihren Kunden anzubieten. DICOM in der Augenheilkunde ist auch längst kein Sonderthema mehr: Weltweit wird die Forderung nach verbesserter Umsetzung des DICOM-Standards mit dem Ziel einer verbesserten Interoperabilität und Konnektivität formuliert (Aaron Y. Lee et al. [1]). Die folgenden Forderungen der American Academy of Ophthalmology sollen hier ausdrücklich unterstrichen werden:

- verbesserte Interoperabilität zwischen den Herstellern (Hard- wie Software),

- maschinenlesbare, auswählbare Reports mit Ausweis strukturierter Messdaten bei bildgebenden Verfahren als auch bei weiteren Verfahren der funktionellen Diagnostik,

- verlustfreie Komprimierung der Pixeloder Voxelwerte bei den Bildrohdaten.

Als Hersteller sollte man nicht warten, bis ein anderer den Anfang macht, sondern sich an die Spitze dieser Initiative stellen. Wir Augenärzte und die uns anvertrauten Patienten werden es danken.

\section{Korrespondenzadresse}

PD Dr. Karsten Kortüm

Prittwitzstr. 43, 89075 Ulm, Deutschland k.kortuem@kortuem.de

Interessenkonflikt. P. Mussinghoff, K. Kortüm, M. Gutfleisch, G. Spital, E. Hansmann, T. Ach, S. Liakopoulos, N. Eter, D. Pauleikhoff und A. Lommatzsch geben an, dass kein Interessenkonflikt besteht. 


\section{Fachnachrichten}

\section{Literatur}

1. Lee AY, Campbell JP, Hwang TS, Lum F, Chew EY (2021) Recommendations for standardization of images in ophthalmology. Ophthalmology 128(7):969-970

2. The Medical Imaging Technology Association (MITA) adoN (2021) DICOM part 3: information object definitions. http://dicom.nema.org/medical/ dicom/current/output/html/part03.html. Zugegriffen: 06.08.2021

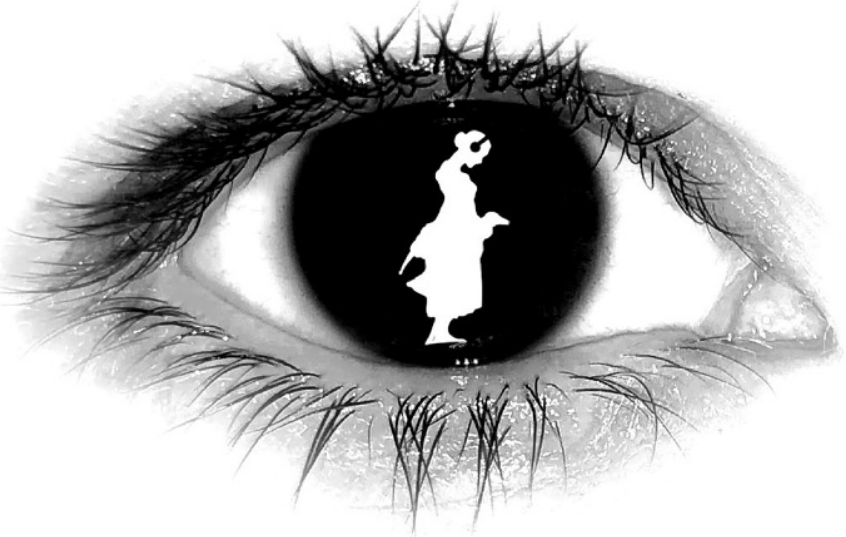

\section{Ophthomania}

Podcast für Augenheilkunde

Hören Sie gerne Podcasts? Haben Sie sich auch schon einmal gefragt, ob es für die Augenheilkunde etwas Kostenfreies in der Form gibt?

Dann begrüßen wir Sie bei Ophthomania, einem Podcast über die Augenheilkunde.

Wir "Patricia, Nina und Mohammed" sind Augenärzte der Uniklinik Göttingen. Wir möchten Ihnen die schöne kleine große Welt der Augenheilkunde etwas näher bringen und versuchen uns daher nun im Podcasten.

Unser Podcast richtet sich an Medizinstudenten/-innen, Assistenzärzte/-innen der Augenheilkunde, medizinisch-informierte interessierte Patienten/-innen und generell an Augenliebhaber/-innen. Alle Angaben haben wir nach bestem Wissen und Gewissen getätigt, wir erheben keinen Anspruch auf Vollständigkeit.

Bereits online zu finden sind die Themen

- Ablatio,

- Makuladystrophien,

- retinaler Venenverschluss

- und Katarakt,

die alle ca. 1-2 Monate um ein weiteres Thema ergänzt werden.

Wir hoffen, unsere Begeisterung und Faszination für diese Fachdisziplin so mit Ihnen teilen zu können und den Funken überspringen zu lassen :-)

Wir wünschen Ihnen viel Spaß!

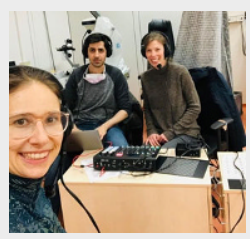

Dr. med. Patricia Take (Assistenzärztin), Dr. med. Nina-Antonia Striebe, FEBO (Funktionoberärztin) \& Dr. med. Mohammed H. Khattab, FEBO (Funktionsoberarzt) an der Augenklinik der UMG Göttingen Kontakt: nina-antonia.striebe@med.uni-goettingen.de

Der Podcast ist auf allen gängigen Plattformen (Spotify, Amazon Music, podigee, Apple Podcasts) sowie hier zu finden: https://augenklinik-goettingen.de/studium/podcast

Über den QR-Code kommen Sie auf eine Auswahl der verschiedenen Plattformen/Webseiten und können direkt loshören! 